\title{
Literature Review on Engineering Design Assessment Tools
}

\author{
Iman Moazzen*; Terry Hansen**; Mariel Miller**; Peter Wild*; Allyson Hadwin**; and LillAnne Jackson* \\ * Faculty of Engineering, University of Victoria, BC, Canada \\ ** Faculty of Education, University of Victoria, BC, Canada
}

\begin{abstract}
Design is one of the twelve graduate attributes that must be assessed as part of the accreditation process for engineering programs in Canada, as required by the Canadian Engineering Accreditation Board (CEAB). However, assessment of design competence is a complex task due to the fact that design process is non-linear and depends on many factors including communication skills, teamwork skills, individual knowledge and skills, and project complexity. Also, for engineering instructors, a key challenge is that this assessment is usually qualitative rather than quantitative. In this paper, a literature review on different design assessment techniques including quantitative, qualitative, and mixed measurement tools is provided, and three key features which must be taken into consideration while developing a design assessment tool are discussed.
\end{abstract}

Keywords: Engineering Design, Assessment, Quantitative Measures, Qualitative Measures

\section{INTRODUCTION}

Design is a central activity to all types of engineering. According to the Canadian Engineering Accreditation Board (CEAB), an accredited program must "culminate in a significant design experience" [6]. In fact, engineering design is a fundamental learning objective for engineering students at all universities [4]. In response to this need, many cornerstone and capstone design courses have been integrated into engineering curriculum ${ }^{1}$. Rigorous assessment of students' design knowledge is crucial to create effective learning environments which facilitate development of design knowledge [4]. Assessment provides valuable feedback for students to evaluate their progress with respect to the intended learning outcomes and become aware of areas in which they may need to improve. Assessment helps educators improve the curriculum and can also help to address public concerns about the quality of educational program [9]. However,

\footnotetext{
1 The terms "cornerstone" and "capstone" refer to the courses taken in the first and senior years, respectively [11].
}

assessment of design competence is a complex task due to the fact that design process is non-linear and depends on many factors including communication skills, teamwork skills, individual knowledge and skills, and project complexity.

In this paper, a literature review of different design assessment tools is provided. This paper is organized as follows: in Section 2, a brief review about the design process is provided. Section 3 summarizes different design assessment tools. Finally, in Section 4 key features of a design assessment tool are discussed.

\section{DESIGN PROCESS}

Different definitions have been presented for the term "design" [10]. In [11] design is articulated as: "engineering design is a systematic, intelligent process in which designers generate, evaluate, and specify concepts for devices, systems, or processes whose form and function achieve clients' objectives or users' needs while satisfying a specified set of constraints."

Three main phases in the design process considered as the common elements in (almost) all engineering design activities are as follows $[6,10]$ :

- Analytical phase which involves identification of need, problem identification, and information gathering

- Creative phase which involves generating conceptual designs and decision making

- Executive phase which involves detailed engineering and implementation

Design knowledge is challenging to learn, teach and assess due to several factors, including:

1- Design process is non-linear and iterative in nature which means that the three aforementioned phases are not necessarily executed one after another, and design experts may iterate between phases several times [10].

2- Design Thinking is described as divergent-convergent questioning. Convergent thinking provides a set of 
answers which hold truth value (facts) for a given question, and is common in the knowledge domain. On the other hand, divergent thinking provides multiple alternative answers regardless of being true or false for a given question, and is common in the concept domain. Design thinking is seen as a series of continuous transformations from the concept domain to the knowledge domain which is difficult to be taught and assessed [11].

3- Design experts can think about system dynamics and anticipate unintended consequences resulting from interaction between different parts. They can also deal with uncertainties and make defensible judgments in ambiguous situations which are very common in design. This advanced thinking is underdeveloped for many university students [11].

4- The language of design plays a key role in engineering design learning and development, but language itself is complex to be evaluated [3].

5- Design is seen as a team process. Typically, good designers are able to function on multi-disciplinary teams [11]. Unfortunately, in most engineering schools teachers mainly focus on technical knowledge. Therefore, students do not receive sufficient training to learn or develop their team skills. Simply placing students in teams without this type of support or training can result in negative experiences and attitudes towards future teamwork make the educational process less effective [1].

6- The design problem is usually open-ended, and the possible solution (final product) may not be universally acceptable because it is one of many possibilities [22]. Also, final product may not reflect individuals' teamwork and communication skills in a group. Thus, while assessment tools often targets the final product, more advanced techniques are needed to capture the whole learning process [23].

\section{DESIGN ASSESSMENT TOOLS}

In this section, available assessment tools are presented. Assessment techniques can be categorized into three main groups: quantitative, qualitative, and mixed. In general, quantitative assessment tools target fewer variables and many cases, and often aim to provide data that support generalizations (breadth) about the phenomenon under study [19]. The data produced by these measurement tools can be easily marked and statistically analyzed. However quantitative tools provide a feasible and cost effective assessment, they are limited in a sense that the numerical description cannot appropriately reflect human perceptions [19]. In contrast, qualitative measures have often been employed in assessment and typically involve fewer cases and many variables. While this type of assessment can be costly and time consuming, it targets at understanding human perception and provides detailed information (depth) about the phenomenon under study [19]. Thus, when both generalization and detailed information are important such as in assessment of design, use of both quantitative and qualitative assessment tools needs to be considered (Figure 1).

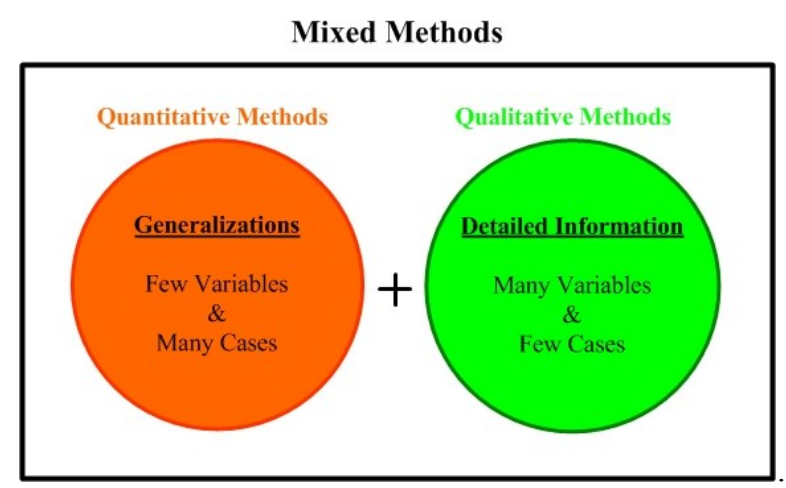

Figure 1. Quantitative, qualitative, and mixed methods

\subsection{Qualitative Assessment Tools}

Three important qualitative assessment techniques are observation, interviews/focus groups, and documents. The key points for each are summarized in Table 1 [19]. In the following section, the application of qualitative data in engineering design assessment is presented.

Table 1. Observation, interviews/focus groups, and documents

\begin{tabular}{|l|l|}
\hline Methods & Key Points \\
\hline Observation & $\begin{array}{l}\text { It is a useful tool to collect data about } \\
\text { the performance or behaviors of groups } \\
\text { or individuals in design activities. The } \\
\text { observer mostly tries to capture key } \\
\text { components of the activities. }\end{array}$ \\
\hline $\begin{array}{l}\text { Interviews/ } \\
\text { Focus }\end{array}$ & $\begin{array}{l}\text { This is a useful tool to collect data that } \\
\text { are less observable such as the } \\
\text { participants' thoughts and perspectives }\end{array}$ \\
\hline Documents & $\begin{array}{l}\text { This includes items such as portfolios, } \\
\text { design reports, free-writing or open- } \\
\text { ended questionnaires. }\end{array}$ \\
\hline
\end{tabular}

\section{A. Observation}

Language is integral to understanding engineering design [3]. One sophisticated tool to assess design language, and accordingly design knowledge, is a verbal protocol in which the participants are monitored and asked to think aloud while working on a design activity. One of the main challenges of using a verbal protocol is to develop a coding scheme that can provide a meaningful interpretation of data $[7,8]$. 
A verbal protocol was used by Bogusch et al. [7] to identify the factors that students think are important to consider while designing a retaining wall system for the Mississippi river. The responses were videotaped and transcribed. A two-dimensional coding scheme was developed to interpret each statement of the recorded data. According to the coding scheme, each statement was assigned to a physical location (wall, water, bank, and shore), and a frame of reference (technical, logistical, natural, and social). Movement from the wall to shore indicates a better and broader understanding of the problem and shows more advanced thinking. Also, movement from technical to social knowledge implies that the students consider more factors in their design and have better understanding of system dynamics. In order to validate the appropriateness of the coding scheme, first year and fourth year students were compared, and a meaningful difference was observed as expected.

Atman et al. [2] also used verbal protocol to compare first-year and fourth year students' design processes. The students were asked to think aloud while working on two ill-structured problems. The design procedure was videotaped, transcribed, and segmented. A coding scheme was designed to categorize each segment to one of the design steps (identifying the need, problem definition, information gathering, modeling, feasibility analysis, evaluation, decision making, communication, and implementation). In order to compare the design processes, the following variables were considered: time spent in each design step, number of transitions between different steps, number of alternative solutions, and solution quality. The findings indicate that fourth year students' design process is more advanced than first year students and fourth year students most likely produce higher quality design solutions.

Atman et al. [3] used both quantitative and qualitative approaches (including verbal protocol) to assess engineering design learning (specifically design language). This mixed approach allowed the researchers to investigate different levels of design understanding. In [3], the main concern was how students (first year and fourth year) use engineering design terminology as they engage in design activities by considering 1) How they select and prioritize design activities by asking students to choose the six most important factors for the design process, 2) How they generate appropriate design language while describing a solution (verbal protocol), and 3) How they demonstrate appropriate planning for information gathering (choosing information from the list that students think are the most important for the design problem), and how well students can apply their knowledge in real design problems. The first year and fourth year students' responses for the first and third tasks (select/prioritize and demonstrate) were compared to design experts. The main goals for this comparison were to investigate the influence of design education on students' design activity priorities and to see if students' priorities increasingly resemble experts' priorities as they go through the engineering program. For the second task (generate), the influence of cornerstone courses on first year students' design language was examined. Students were asked to think aloud while solving a design problem once at the beginning of the design course (pre-test) and again at the end (post-test). To interpret data, a coding scheme was developed based on the expected behaviours and design activities/stages. The comparison showed that pre-test responses were most solution-focused while post-test responses were processoriented which is indicative of more advanced thinking.

\section{B. Interview}

Assessing students' sophistication of thinking or depth of processing is a valuable aspect of assessing engineering design. Interviews have also been used to gain insight into students' thinking of open-ended problems. In particular, some research has used the Perry model which suggests that students' cognitive processes develop from simple black/white thinking (right or wrong answer) to a more complex evaluation of alternatives [20] (Figure 2).

Pavelich and Moore [24] conducted hour-long, structured interviews with different groups of students (first, second, and fourth year) to evaluate the Perry model level. The structured interview consists of leading and follow-up questions (to encourage students to provide more details about their thinking and justify their points of view). Statistical and verbal evaluation methods were used to analyze recorded data and Perry's level. The Perry model was also used in [20] in the same way (interview) to examine the influence of a first-year design course on engineering students' intellectual development. Findings from both studies indicate that the design courses clearly impact intellectual development.

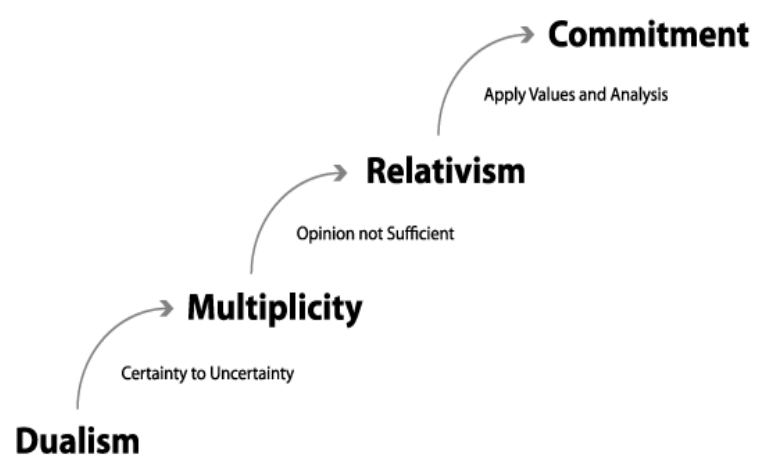

Figure 2. Nelson's Adaptation of the Perry Framework [29] 


\section{Documents}

Documents or artifacts can include many items such as writing, concept maps, and portfolios [22]. One of the main challenges of using documents is to develop appropriate marking rubrics. Two common scoring rubrics are analytic and holistic. An analytic rubric uses a separate score for different aspects of students' responses while a holistic rubric uses a single score to evaluate the whole process [19].

Baily et al. used analytical writing to assess design knowledge by asking students to critique two ill-structure design processes [4]. Analytic writing is process-focused, and it's a powerful tool because of its ability to evaluate students' knowledge in almost all levels of Bloom's taxonomy (remembering, understanding, applying, analyzing, evaluating, and creating [4]). The analytic marking rubric was developed in [4] based on three main phases of design process (problem formulation, problem solving, solution implementation). Writing (reflective essay) was also used as an assessment tool in [9]. Another form of writing called free writing (write non-stop with no gaps for five to ten minutes) was used in [22]. In that study, students were requested to do freewriting about a given design problem at different times of a design activity (start, middle way, and the end). By comparing the results, the general design knowledge and students' progress can be evaluated.

Portfolios are potentially useful for assessment due to the fact that they can be integrated into class activities. In fact, the contents of portfolio are already natural products of a design course, not add-ons. As articulated in [22]: "Instead of a one-off portrait of a student on a particular day, they provide a more equitable and fair representation of student work over time." A common portfolio should include 1) student work, both drafts and finished products, over a period of time, 2) three or four of their best polished pieces [22].

According to semantic memory theory, knowledge is stored in a network format where concepts are connected to each other [27]. Based on this premise, concept maps can be used to assess knowledge. Concept maps consist of nodes representing important concepts and labeled lines showing relation between concepts. The application of concept maps for both course-level and program-level assessment in engineering education is discussed in [27]. Concept maps are used in [22] to specifically assess design knowledge where students were asked to draw a concept map about the design process at the beginning and end of first-year design courses. Although concept maps can effectively capture the student's level of integrated knowledge, they are difficult to be scored which limits their feasibility as an assessment tool. This issue has been addressed in [26]. Traditional scoring typically involves counting three map features: concepts, hierarchies (the levels that extend from the central concept), and cross-links (number of links between established hierarchies). One scoring method proposed in [26] considers comprehensiveness, organization, and correctness. A comprehensive study about concept maps can be found in [28].

In order to ensure that findings from qualitative research are reliable, some methods such as triangulation and member checking should be considered to establish trustworthiness (see [19] and references there).

\subsection{Quantitative Assessment Tools}

Two common quantitative methods are surveys and questionnaires.

Gentili et al. used a survey to provide a quick and reliable feedback mechanism [15]. Seven categories in the survey include teamwork and communication skills, and the five fundamental elements of design process (according to of Transferable Integrated Design Engineering Education). For each category, several components are considered to provide a detailed description. Students are able to use the survey to assess their perception of class emphasis in each area along with their perceived growth during the course.

A questionnaire called CADEK (Comprehensive Assessment of Design Engineering Knowledge) was developed in [23]. The instrument consists of 20 constructed-response questions designed to assess students' design skills and concepts (design process, teamwork, and communication). In order to validate the tool, after each question, students were asked to indicate if the question was appropriate to measure learning in the course.

\section{KEY FEATURES OF A GENERAL ASSESSMENT TOOL}

In general, a good assessment tool should have three key features: systematic, flexible, and time and cost efficient.

Systematic: To maximize the consistency and minimize the problem reliability with raters, the assessment technique should be systematic. An example of a systematic approach was proposed in [9] to assess three educational objectives defined as design process, teamwork and communication. In order to track students' progress, formative (midway) and summative (end-of- 
program) assessments were considered. The mid-program assessment is a mixed approach combining short-answer quiz, in-class group activity, and reflective essay, and it mainly focuses on early stages of Bloom's taxonomy (the ability to understand the concepts, and use them to solve a particular problem). On the other hand, end-of-program assessment is mainly based on documentation and focuses on the quality of design products. The formative assessment is relatively short, but the summative assessment needs one or more academic terms.

Flexibility: Since the projects in design courses differ greatly in breadth (open-ended problems which require significant work on the early stages of design process i.e. identification of need \& problem definition and information gathering) and depth (problems are relatively well-defined and students focus mainly on the last stages of design process i.e. detailed engineering and implementation), a good assessment technique needs to be flexible to be applicable to both. A good example of such an approach can be found in [6].

Time and cost efficient: In order to track student's progress through the engineering program, assessing first year students' design knowledge is very crucial. In many engineering schools, the number of first year students is very large, and assessing large classes have always been challenging [21]. Frank et al. proposed a paper-based design skill assessment (DSA) instrument which can be applied to large number of students in a short time, marked even with non-experts in design, and applied to all design courses [12-14]. "Pre-activity" and "Postactivity" assessments (beginning and end of the design courses) were considered in which the students were requested to write about the design process that they would consider in order to solve an open-ended problem. Analytical marking rubrics were developed to evaluate student's design knowledge.

\section{CONCLUSION}

In this paper, different engineering design assessment tools are summarized. Most engineers are comfortable with numbers and accordingly they prefer quantitative methods. Depending on the purpose of the investigation, however, qualitative assessment techniques or even a combination of both qualitative and quantitative techniques may yield a more comprehensive analysis of the engineering design context. Sometimes, a quantitative study can reveal a need for a qualitative research and vice versa (refer to [19] and references there for more information).

\section{ACKNOWLEDGEMENTS}

The authors greatly acknowledge funding from NSERC, Canada.

\section{REFERENCES}

[1] Adams, S. and Okudan, G., "A comprehensive model for student design team formation and performance in the engineering classroom", International Conference on Engineering Education, 2003, Valencia, Spain.

[2] Atman, C.J., Cardella, M.E., Turns, J., and Adams, R., "Comparing freshman and senior engineering design processes: an in-depth follow-up study", Elsevier, 2004.

[3] Atman, C.J., Deborah Kilgore, and Ann McKenna. "Characterizing Design Learning: A Mixed-Methods Study of Engineering Designers' Use of Language," Journal of Engineering Education, vol. 97, no. 3, pp. 309-326, 2008.

[4] Bailey, R., and Szabo, Z., "Assessing engineering design process knowledge". International Journal of Engineering Education, Vol. 22, No. 3, pp. 508-518, 2006.

[5] Bailey, R., Szabo, Z., and Sabers, D. "Assessing Student Learning about Engineeering Design in Project-Based Courses". Proceedings of ASEE Conference \& Exposition, pp. 753-766, 2004

[6] Bazylak, J., and Wild, P., "Evaluation system for capstone engineering design reports", 2010.

[7] Bogusch, L. L., Turns, J., and Atman, C. J., "Engineering design factors: how broadly do students define problems?" ASEE/IEEE Frontiers in Education Conference S3A-7, 2000.

[8] Chi, M. T. H. "Quantifying Qualitative Analyses of Verbal Data: A Practical Guide." Journal of the Learning Sciences, pp. 271-315, 1997.

[9] Davis, D.C., K.L. Gentili, M.S. Trevisan, and D.E. Calkins., "Engineering Design Assessment Processes and Scoring Scales for Program Improvement and Accountability." Journal of Engineering Education, pp. 211-221, 2002.

[10] Dym, C.L., Engineering Design: A synthesis of views, Cambridge University Press, 1994.

[11] Dym, C.L., Agogino, A.M., Eris, O., Frey, D.D., and Leifer, L.J., "Engineering design thinking, teaching, and learning", Journal of Engineering Education, pp. 103-120, 2005.

[12] Frank, B., and Strong, D., "Survey-based assessment of design skill development in engineering project courses", 2008.

[13] Frank, B., and Strong, D., "Development of a Design Skill Assessment Tool", 2010.

[14] Frank, B., and Strong, D., Boudreau, J., and Pap, A. "Design skill assessment from pre-university to third year", 2009. 
[15] Gentili, K., Lyons, J., Davishahl, E., Davis, d., and Beyerlein, S., "Measuring added-vaalue using a team design skills growth survey", 2005.

[16] Kadlowec, J., Bhatia, K., Chandrupatla, T. R., Chen, J. C., Constans, E., Hartman, H., et al. "Design integrated in the mechanical engineering curriculum: Assessment of the engineering clinics." Journal of Mechanical Design, 2007.

[17] Ku, W. A., "Using Concep Maps to Explore the Conceptual Knowledge of Technology students: An Exploratory study", 2007.

[18] Lewis, P., Aldridge, D., and Swamidass, P. M., “Assessing Teaming Skills Acquisition on Undergraduate Project Teams" Journal of Engineering Education, 1998.

[19] Leydends, Moskal, \& Pavelich, "Qualitative methods used in the assessment of engineering education", Journal of Engineering Education, 2004.

[20] Marra, R. M., Palmer, B., and Litzinger, T. A. "The Effects of a First-Year Engineering Design Course on Student Intellectual Development as Measured by the Perry Scheme", Journal of Engineering Education, 2000.

[21] Mehta, S. I., and Schlecht, N. W. "Computerized Assessment Technique for Large Classes", Journal of Engineering Education, 1998.

[22] Newstetter, and Khan, "A developmental approach to assessing design skills and knowledge", 1997.
[23] Okudan, G., Ogot, M., Zappe, S., and Gupta, S. "Assessment of learning and its retention in the engineering design classroom", American Society for Engineering Education, 2007.

[24] Pavelich, and Moore, "Measuring the effect of experiential education using the Perry Model", The Journal of Engineering Education, 1996.

[25] Puente, S.M.G., M. van Eijck, M.V., and Jochems, W., "Towards characterising design-based learning in engineering education: a review of the literature", European Journal of Engineering Education, 2011.

[26] Sacre, M. B., Gerchak, J., and Lyons, M. "Scoring Concept Maps: An Integrated Rubric for Assessing Engineering Education", Journal of Engineering Education, 2004.

[27] Turns, J., Atman, C. J., and Adams, R. "Concept Maps for Engineering Education: A Cognitively Motivated Tool Supporting Varied Assessment Functions", IEEE Transactions on Education, 2000.

[28] Wendy A. Ku., Using concept maps to explore the conceptual knowledge of technology students: An exploratory study. PhD Dissertation, The Ohio State University, 2007.

[29] http://wiki.ubc.ca/Documentation:Guide_to_Teaching_for _New_Faculty_at_UBC/Resource_1:_How_People_Learn 